\title{
RECENT PROGRESS IN ELECTRON CYCLOTRON RADIATIVE TRANSPORT MODELLING OF FUSION PLASMAS IN VIEW OF ITER AND DEMO APPLICATIONS
}

\author{
F. ALBAJAR ${ }^{a}$, M. BORNATICI ${ }^{b}$, F. ENGELMANN ${ }^{c}$ \\ ${ }^{a}$ Asociación EURATOM-CIEMAT, Madrid, 28040, Spain \\ ${ }^{b}$ Physics Department “A. Volta”, University of Pavia, Pavia, 27100, Italy \\ ${ }^{\circ}$ Max-Planck-Institut für Plasmaphysik, Garching, 85748, Germany
}

\begin{abstract}
As it was recognised that local electron cyclotron (EC) wave power losses can be a competitive contribution to the $1 \mathrm{D}$ electron power balance for reactor-grade tokamak plasmas in regimes as anticipated for steady-state operation, a systematic effort is ongoing to improve the modelling capability for the radial profile of EC wave emission. This effort aims at generating a hierarchy of codes that cover the non-local behaviour of EC wave transport with good accuracy and also provide sufficient computational efficiency for being usable in 1D transport studies. An overview on this activity is given with emphasis on the code RAYTEC which explicitly addresses the geometrical effects present in toroidal plasmas with arbitrary cross-section.
\end{abstract}

\section{Introduction}

Electron cyclotron (EC) wave emission has been shown to be a significant contribution to the local electron power balance of high temperature fusion magnetoplasmas with core electron temperatures of about $35 \mathrm{keV}$ or higher as anticipated for steady-state operation scenarios of ITER and DEMO ${ }^{1}$. The estimation of the level of EC wave emission in system and 1D transport studies is usually carried out by using approximate models derived from the global EC emission ${ }^{2,3}$ or routines like CYTRAN ${ }^{4}$ and CYNEQ ${ }^{5}$ which, to some extent, incorporate wave transport effects, but assume isotropy of the radiation field.

In order to improve the modelling capability for the profile of the net EC wave power density $d P / d V$ locally emitted (or absorbed), as a first step, recently the accuracy of the Trubnikov ${ }^{6}$ and Robinson ${ }^{7}$ formulae for the wave absorption coefficients was analysed ${ }^{8}$ and their practical suitability for calculating $d P / d V$ was investigated ${ }^{9}$, adopting the code EXACTEC which is based on an exact solution of wave transport for a cylindrical plasma with circular cross section and specular wall reflection ${ }^{10}$. As a result, Robinson's formula was found to provide, overall, both good accuracy and numerical efficiency in modelling hot fusion plasmas. 
As a second step, a wave transport model analysis has been made for a circularly cylindrical fusion plasma ${ }^{11}$ comparing CYTRAN and CYNEQ with EXACTEC and former results from SNECTR ${ }^{12}$. As expected, the assumption of the wave intensity being isotropic made in the former models tends to be a good one if wall reflection is strong and diffuse. Hence, CYTRAN and CYNEQ are fast routines well-suited for transport simulations of fusion plasmas. On the other hand, EXACTEC has confirmed earlier results from SNECTR for specular reflection and in situations in which wall reflection has a diffusive component and, hence, reduces the anisotropy of the radiation field, provides a lower bound (not far from the actual values) to the net EC power density emitted from the hot plasma core as well as to re-absorption in the cool edge plasma.

As a third step, to cover the effects of a non-circular plasma cross-section and of toroidicity, a new ray tracing code, called RAYTEC, is being developed. The motivation for this work is to provide an alternative to SNECTR which is no longer in active use. Presently, the code addresses specular reflection at the wall which allows tracing rays without having to rely on Monte-Carlo methods (as does SNECTR). The code, primarily intended to be applied to plasmas in thermodynamic equilibrium, can readily be extended to include a fast electron population described by a Maxwellian distribution (not shifted with respect to the bulk electron distribution). The efficiency of the computations has been enhanced by adopting Robinson's formula for the calculation of the EC absorption coefficient as well as by an appropriate modelling of the inner hot plasma for the lower frequencies for which the plasma effectively behaves as a black body and which contributes little to $d P / d V$.

In this paper, we concentrate on describing the approach adopted in RAYTEC and report on first results obtained with this code for reactor-grade plasmas.

\section{The RAYTEC approach}

For plasmas in thermodynamic equilibrium, the net EC wave power per unit volume radiated at the position $\mathbf{r}$ is

$$
\frac{d P(\mathbf{r})}{d V}=\sum_{\sigma=\mathrm{X}, \mathrm{O}} \int_{\omega_{\min }^{\sigma}}^{\infty} \mathrm{d} \omega \int d^{2} \Omega_{\hat{s}} \alpha^{\sigma}(\mathbf{r}, \omega, \hat{\mathbf{s}})\left[I_{\mathrm{bb}}^{\sigma}(\mathbf{r}, \omega)-I^{\sigma}(\mathbf{r}, \omega, \hat{\mathbf{s}})\right]
$$

with $\omega$ the wave frequency, $\sigma=\mathrm{X}, \mathrm{O}$ the extraordinary and ordinary polarization modes, $d^{2} \Omega_{\hat{s}}$ the element of solid angle around the ray direction $\hat{\mathbf{s}}, \alpha$ is the absorption coefficient, and $I_{b b}^{\sigma}(\mathbf{r}, \omega) \equiv\left(N_{r}^{\sigma}\right)^{2} \omega^{2} T(\mathbf{r}) / 8 \pi^{3} c^{2}$ the black-body intensity for the (local) electron temperature $T(\mathbf{r})$ and a (local) ray refractive 
index $N_{r}^{\sigma}$. In Eq.(1), the specific radiation intensity $I^{\sigma}$ is governed by the (steady-state) radiative transfer equation (RTE), see, e.g., ${ }^{2,}{ }^{10}$, which in the context of geometrical optics accounts for the non-local nature of the radiation process that is due to both re-absorption of radiation and reflection at the wall surrounding the plasma. An analytical solution for $I^{\sigma}$ was obtained for a system having (circularly) cylindrical symmetry and assuming that the reflection at the wall is specular ${ }^{10}$. In toroidal geometry, the poloidal symmetry of the radiation intensity is lost due to (i) the inhomogeneity of the toroidal magnetic field along the horizontal coordinate which enhances emission and absorption at the highfield side of the plasma, (ii) the impact on the ray path length, which affects the effective optical thickness of the plasma, and (iii) the variation of the angle of propagation relative to the magnetic axis along the ray path. Elongation of the plasma cross-section further enhances the latter two effects and also causes wall reflection to reduce the anisotropy of the radiation field.

For arbitrary geometry and plasma profile, integration of the RTE from a point $\mathbf{r}_{w, 1}$ at the wall to an arbitrary point $\mathbf{r}$ within the plasma along a ray yields

$$
\frac{I(s(\mathbf{r}))}{N_{\mathrm{r}}^{2}}=I_{\mathrm{ref}}\left(s\left(\mathbf{r}_{\mathrm{w}, 1}\right)\right) e^{-\tau\left(s(\mathbf{r}), s\left(\mathbf{r}_{\mathrm{w}, 1}\right)\right)}+i\left(s(\mathbf{r}), s\left(\mathbf{r}_{\mathrm{w}, 1}\right)\right)
$$

where

$$
i\left(s(\mathbf{r}), s\left(\mathbf{r}_{\mathrm{w}, 1}\right)\right)=\frac{\omega^{2}}{8 \pi^{3} c^{2}} \int_{s\left(\mathbf{r}_{\mathrm{w}, 1}\right)}^{s(\mathbf{r})} d s^{\prime} T\left(s^{\prime}\right) \alpha\left(s^{\prime}\right) e^{-\tau\left(s(\mathbf{r}), s^{\prime}\right)},
$$

$\tau$ is the optical distance along the ray path, and $s$ is a coordinate along the ray trajectory (the dependencies on $\sigma, \omega$, and $\hat{\mathbf{s}}$ have been dropped for convenience). In Eq.(2), $I_{\text {ref }}$ refers to the radiation intensity after reflection. For specular wall reflection, the incident and reflected intensities at the point $\mathbf{r}_{w, 1}$ are related by

$$
I_{\text {ref }}\left(s\left(\mathbf{r}_{\mathrm{w}, 1}\right)\right)=R_{\mathrm{w}} I_{\mathrm{inc}}\left(s\left(\mathbf{r}_{\mathrm{w}, 1}\right)\right)
$$

(disregarding polarization scrambling, for simplicity) where $R_{\mathrm{w}}$ is the (effective) wall reflection coefficient $\left(0 \leq R_{\mathrm{w}} \leq 1\right)$. The two terms of the right-hand side of Eq.(2) describe the re-absorption and the (effective) emission of radiation over the ray path between $\mathbf{r}_{\mathrm{w}}$ and the investigated position $\mathbf{r}$.

By applying Eqs.(2) and (4) in a recurrent way between two consecutive reflection points $\mathbf{r}_{\mathrm{w}, \mathrm{k}}$ and $\mathbf{r}_{\mathrm{w}, \mathrm{k}+1}$ on the ray path as illustrated in Fig.1, in backward direction over $n(n \geq 2)$ reflections, yields 


$$
\begin{aligned}
I_{\text {ref }}(1)= & R_{\mathrm{w}}^{\mathrm{n}-1} I_{\mathrm{ref}}(n) \exp \left(-\sum_{k=1}^{n-1} \tau(k, k+1)\right) \\
& +\sum_{k=2, n-1} R_{\mathrm{w}}^{\mathrm{k}} i(k, k+1) \exp \left(-\sum_{j=1}^{k-1} \tau(j, j+1)\right)+R_{\mathrm{w}} i(1,2)
\end{aligned}
$$

with $i(k, k+1)$ given by applying Eq.(3) to $\mathbf{r}_{\mathrm{w}, \mathrm{k}}$ and $\mathbf{r}_{\mathrm{w}, \mathrm{k}+1}$. Note that the ray direction $\hat{\mathbf{s}}$ is changed in every reflection depending on the geometry of the first wall (or, more generally, on the local properties of the wall at the reflecting point). For a sufficiently high number $n$ of reflections, the optical length of the ray becomes much larger than one, $\sum_{k=1}^{n-1} \tau(k, k+1) \gg 1$, and the specific intensity does no longer depend on its initial value which then can be taken $I_{\text {ref }}(n)=0$, for simplicity. The number of reflections to be considered depends on the plasma dimension and parameters (temperature, density and magnetic field), but also on the wave frequency and ray direction. Specifically in an optically thick situation, a small number of reflections already provides a good estimate of $I_{\text {ref }}(1)$.

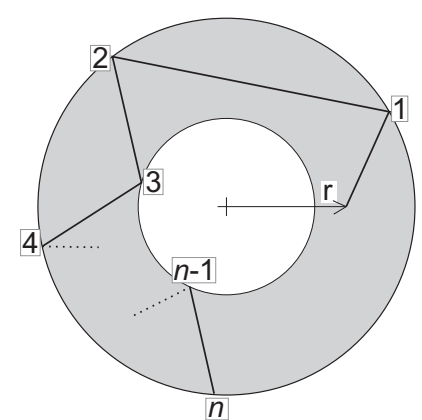

Figure 1. Schematic view of a (straight) wave trajectory in the equatorial plane of a plasma torus.

The described method to calculate the specific intensity for an arbitrary plasma and first-wall geometry has been implemented in the RAYTEC code. In contrast to the approach followed in SNECTR ${ }^{12}$ in which the net power density radiated is calculated from the difference between the radiation intensity emerging from and entering through the surface of a given plasma region when launching $\mathrm{n}$ random rays from the plasma wall to the inner plasma, in RAYTEC $d P / d V$ is evaluated at every plasma position $\mathbf{r}$ using the exact expression (1).

The efficiency of the computation in the RAYTEC code has been enhanced by adopting Robinson's formula for the calculation of the EC absorption coefficient $^{7,8,9}$ and by an appropriate modelling in the inner hot plasma for the low frequencies for which the plasma is a black body. In fact, the computer time 
needed to calculate the local net EC power loss in the low-frequency part of the wave spectrum, contributing little to $d P / d V$, is significantly longer than for higher frequencies. This is due to the slow convergence of the integration in Eq.(1) for low frequencies where emission exceeds absorption only marginally and, in addition, the spectrum has a line-like structure. For these low frequencies, in RAYTEC the diffusion limit of radiative transfer, see, e.g., ${ }^{10}$, is used for which one has

$$
\frac{d^{2} P^{\sigma}}{d V d \omega}(\mathbf{r}) \simeq-\left(N_{r}^{\sigma}\right)^{2} \frac{\omega^{2}}{8 \pi^{3} c^{2}}\left(\int d^{2} \Omega_{\hat{s}} \frac{\hat{\mathbf{s}} \hat{\mathbf{s}}}{\alpha^{\sigma}(s(\mathbf{r}), \omega, \hat{\mathbf{s}})}\right): \nabla \nabla T(\mathbf{r}) .
$$

This approximation is valid for the frequency range

$$
\omega_{\min }^{\sigma}(\hat{\mathbf{s}}) \leq \omega \leq \omega_{b b}^{\sigma}(\hat{\mathbf{s}})
$$

where $\omega_{\min }^{\sigma}$ is the lower limit of the $\omega$-integration in Eq.(1) and $\omega_{b b}^{\sigma}$ is the upper bound of the frequency range for which the plasma effectively radiates as a black-body determined by $\omega_{b b}^{\sigma}(\hat{\mathbf{s}})=\min \left\{\omega_{1}^{\sigma}(\hat{\mathbf{s}}), \omega_{2}^{\sigma}(\hat{\mathbf{s}})\right\}$ with, respectively, $\omega_{1}^{\sigma}(\hat{\mathbf{s}})$ and $\omega_{2}^{\sigma}(\hat{\mathbf{s}})$ the upper limit of the frequency range for which the optical distance from the wall is large, $\tau^{\sigma}(\omega, \hat{\mathbf{s}}) \gg 1$, and that for which the radiative mean free path $(1 / \alpha)$ is short compared to the scale-length of the temperature variation, $\left|\frac{1}{\alpha^{\sigma}(s(\mathbf{r}), \omega, \hat{\mathbf{s}})} \frac{1}{T} \frac{d T}{d s}\right| \ll 1$. Note that the frequency range (7) depends on both the mode $\sigma$ and the ray direction. Typical values of the frequency limit $\omega_{b b}^{\sigma}(\hat{\mathbf{s}})$ for the high-temperature ITER case with an advanced $T$ profile, analysed in the following section are 3.5-4.5 times the EC frequency $\omega_{\mathrm{c}}$ for the extraordinary mode $(\sigma=\mathrm{X})$ and $(2-3) \omega_{\mathrm{c}}$ for the ordinary mode $(\sigma=\mathrm{O})$ as seen in the left part of Fig.2 where the frequency spectrum of both the specific intensity $I$ and (in the right part) the net EC radiative power density $d^{2} P / d V d \omega$ are shown; from the latter it is transparent that the contribution from the frequency range (7), that is $\omega_{b b}^{\mathrm{o}}(\hat{\mathbf{s}}) \lesssim 2.5 \omega_{c}$ and $\omega_{b b}^{\mathrm{x}}(\hat{\mathbf{s}}) \lesssim 4.2 \omega_{c}$, to $d P / d V$ is, in fact, negligibly small.

The low-frequency waves travelling in a toroidal plasma towards the highfield side are cut in RAYTEC when approaching the cut-off frequency. This treatment has been shown to be robust compared to other approximations. The contribution of these waves to $d P / d V$ is likewise negligible.

The RAYTEC code has been validated against the exact code EXACTEC in the limit of (circularly) cylindrical plasmas producing identical results. 

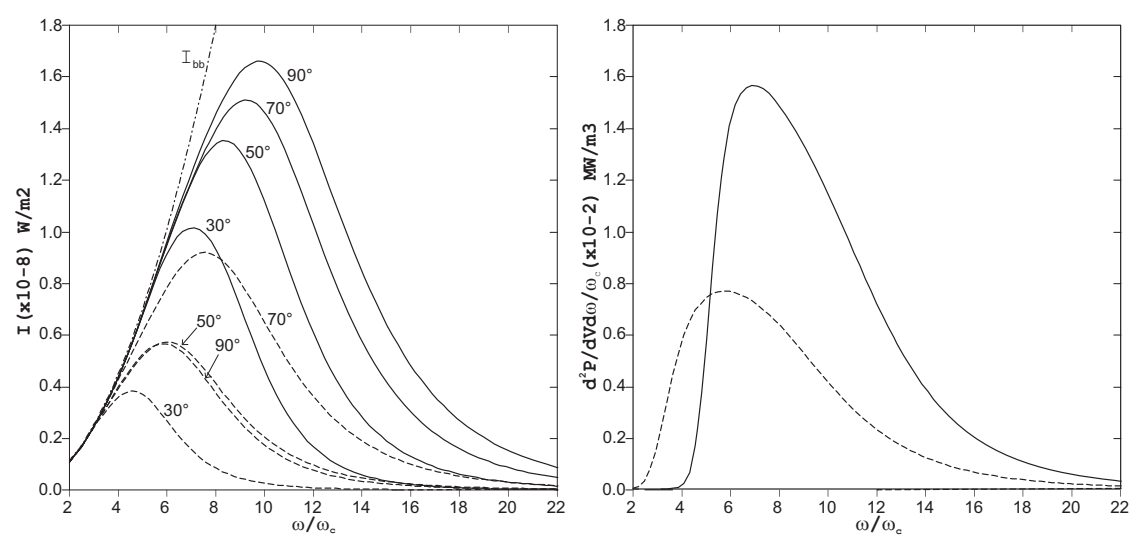

Figure 2. Frequency spectrum of the specific intensity $I$ (left plot) for horizontal ray paths with various angles $\left(30^{\circ}, 50^{\circ}, 70^{\circ}\right.$ and $\left.90^{\circ}\right)$ relative to the magnetic field, and of the net $\mathrm{EC}$ radiative power density $d^{2} P / d V d \omega$ (right plot) in the plasma core $(\rho=\mathrm{r} / a=0.05)$ : X-mode (solid curves), Omode (dashed curves); in the left plot the black-body intensity is also shown (dot-dashed curve); ITER-like plasma parameters, advanced $T$ profile (see Sect.3) and a wall reflectivity $R_{\mathrm{w}}=0.8$ are taken.

\section{Analysis of ellipticity and toroidicity effects}

RAYTEC has been used to analyse the impact of (vertical) plasma elongation and toroidicity. For this, ITER-like parameters have been adopted, namely, a plasma minor radius $a=2 \mathrm{~m}$, a major radius $R=6.2 \mathrm{~m}$, that is an aspect ratio $\mathrm{A}_{\text {ref }}=R / a=3.1$, elongation $\kappa_{\text {ref }}=1.7$ and a toroidal magnetic field $\mathrm{B}=5.3 \mathrm{~T}$ on the magnetic axis. For simplicity, the magnetic surfaces, characterized by the normalized radius coordinate $\rho=r / a$, were assumed to be concentric ellipses. The profiles of the electron density $n$ and temperature $T$ have been taken in the form $n(\rho)=n(0)\left(1-\rho^{2}\right)^{\gamma_{n}}$ with a peak density $n(0)=1.1 \times 10^{20} \mathrm{~m}^{-3}$ and $\gamma_{\mathrm{n}}=0.1$, giving a volume average density of about $10^{20} \mathrm{~m}^{3}$, and $T(\rho)=(T(0)-T(1))\left(1-\rho^{\beta_{T}}\right)^{\gamma_{T}}+T(1)$. Two temperature profiles with a peak temperature $T(0)=45 \mathrm{keV}$ and a volume average temperature of about $18 \mathrm{keV}$ were considered: a profile flat in the core plasma with a steep gradient around $\rho=0.6$ which simulates steady-state operation conditions $\left(\gamma_{\mathrm{T}}=8.0, \beta_{\mathrm{T}}=5.4\right.$; in the following referred to as 'advanced' profile $)$ and a 'parabola-like' one (characterized by $\gamma_{\mathrm{T}}=1.5, \beta_{\mathrm{T}}=2.0$ ). The wall reflection coefficient has been taken $R_{\mathrm{w}}=0.8$.

The radial profiles of the net EC power emitted, $d P / d V$, obtained from the RAYTEC code are shown in Fig.3 for the reference case of plasma elongation $\kappa_{\text {ref }}=1.7$, 'advanced' $T$ profile, and different poloidal angles $\theta$. The angle is counted counterclockwise from the horizontal pointing towards the low-field 
side. As expected, at a given $\rho$, in the inner plasma $d P / d V$ increases from $\theta=0$ to $\theta=\pi$, that is towards higher fields the EC emission is enhanced, the enhancement being maximum for intermediate plasma radii, $0.3 \leq \rho \leq 0.4$ : $d P / d V$ for $\theta=\pi$ is about twice the one for $\theta=0$. From Fig. 3 it is also seen that the profile reversal occurring in the edge plasma due to the accumulation of radiation caused by wall reflection, is significantly enhanced on the high-field side. The degree of increase of $d P / d V$ with poloidal angle is slightly reduced around the vertical direction, $\theta=\pi / 2$, due to an increase of the effective plasma dimension connected with elongation. Fig. 3 shows that the $d P / d V$ profile averaged over poloidal angles, closely follows the one for $\theta=\pi / 2$, the extrema of the former being somewhat more pronounced. Similar results have been obtained for other plasma parameters and temperature profiles.
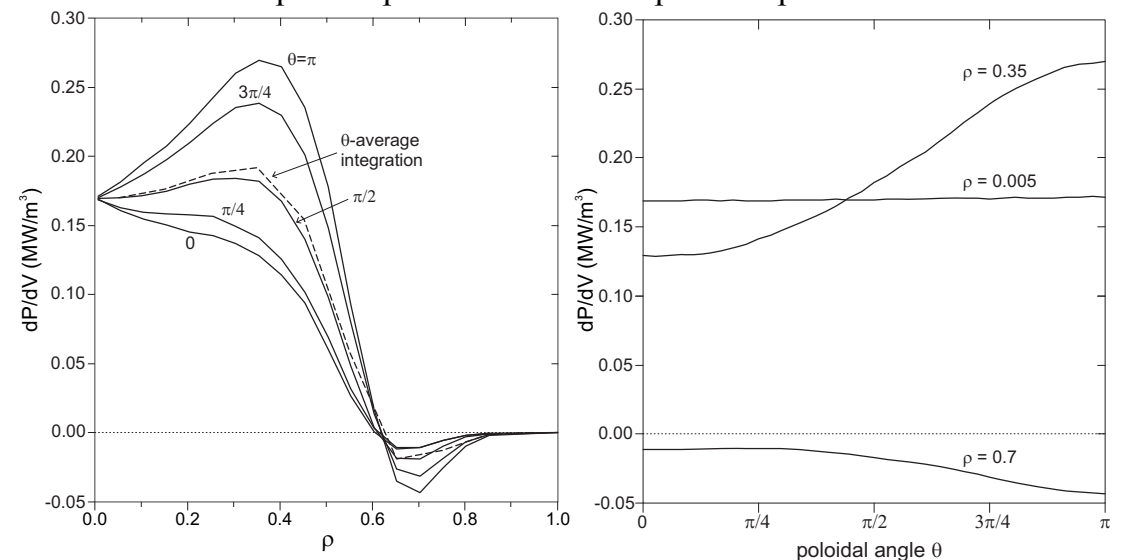

Figure 3. Left plot: radial profile of the EC radiative power density $d P / d V$ from RAYTEC for toroidal geometry $\left(\mathrm{A}_{\mathrm{ref}}=3.1\right)$, the reference elongation $\kappa_{\mathrm{ref}}=1.7$ and an advanced $\mathrm{T}$ profile, for various poloidal angles $\theta$, as well as the poloidal average of $d P / d V$ (dashed curve); right plot: the dependence of $d P / d V$ on the poloidal angle for three radial positions: in the plasma core $(\rho=0.005)$, at an intermediate radius $(\rho=0.35)$, and in the range of profile reversal $(\rho=0.7)$.

In the following, only the poloidally averaged $d P / d V$ profiles which are the ones of relevance for 1D transport studies, will be further discussed. Figs. 4(a) and 4(b) illustrate the results for $\mathrm{A}_{\text {ref }}=3.1$ and different values of elongation $\left(\kappa=1,1.35,2.05\right.$ as well as $\left.\kappa_{\text {ref }}=1.7\right)$ for the advanced and the parabola-like temperature profiles. To keep the plasma volume constant in this comparison, an effective minor radius $a_{\text {eff }}(\kappa)=a \sqrt{\kappa_{\text {ref }} / \kappa}$ is used. In addition, in order to eliminate the effect of a varying aspect ratio $\mathrm{A}$ in the analysis of the impact of ellipticity, an effective major radius $R_{\text {eff }}$ is adopted such that the aspect ratio $\mathrm{A}=R_{\text {eff }} / a_{\text {eff }}$ remains constant equal to $\mathrm{A}_{\text {ref }}=3.1$. In Figs.4, the results from a 
specific ray tracing code for cylindrical geometry and elliptical cross-section are also shown. For the limiting case of circular cross-section $(\kappa=1)$ the exact result as obtained from EXACTEC is also plotted, showing a perfect agreement with the one from the ray tracing code.
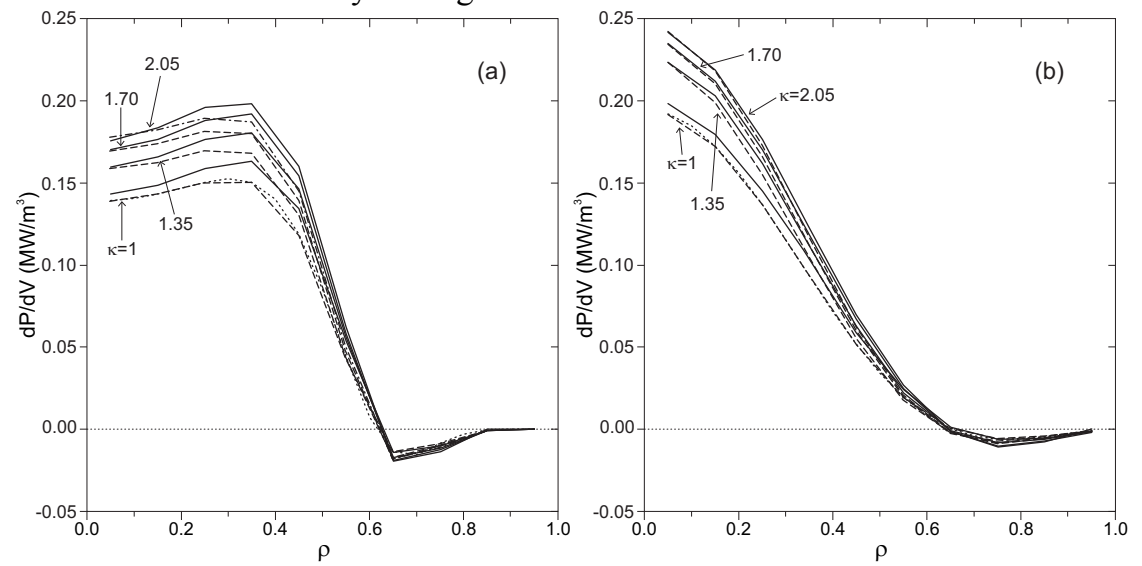

Figure 4. Poloidally averaged net EC wave power density $d P / d V$ from RAYTEC for $\mathrm{A}_{\text {ref }}=3.1$ and "advanced" (left plot (a)) and parabola-like (right plot (b)) temperature profiles, for various values of plasma elongation; for comparison, the results for cylindrical geometry (dashed) and the limiting case of a circularly cylindrical plasma calculated with EXACTEC (dotted) are also shown.

From Figs. 4 it is seen that in the core plasma $d P / d V$ increases with elongation by about $25 \%$ when going from the circular plasma to the highly elongated case $\kappa=2.05$. In the cooler outer layers elongation tends to enhance the profile reversal. This trend is due to the growing isotropisation of the specific intensity for increasing elongation of the plasma cross-section: whereas for a circular plasma, the EC wave power emitted by the hot inner plasma, upon reflection, is strongly concentrated onto the hot core and re-absorbed there, this symmetry effect weakens for increasing elongation and a larger part of the reflected wave power is absorbed in the intermediate and edge plasma. This tendency is enhanced for increasingly stronger wall reflection.

A similar effect is observed when going from a cylindrical plasma to toroidal geometry. As seen in Fig.4, whereas the values of $d P / d V$ at the plasma centre are almost unchanged, toroidicity, and in particular the inhomogeneity of the magnetic field enhances $d P / d V$ for intermediate radii.

The dependence of $d P / d V$ on aspect ratio A for a central $(\rho=0.05)$ and an intermediate $(\rho=0.4)$ plasma position is shown in Fig.5 for the two $T$ profiles and two plasma elongations, $\kappa=1$ and $\kappa_{\text {ref }}=1.7$. From this figure it is seen that (i) $d P / d V$ increases with the elongation for a given value of $\mathrm{A}$, as already 
observed in Figs.4 and (ii) the toroidal effects, mainly the correction to the ray path length, effectively decrease the optical distance of the inner plasma from the wall with decreasing A so that $d P / d V$ slightly increases. The inhomogeneity of the toroidal magnetic field enhances the geometrical effect for intermediate ( $\rho \approx 0.4$ ) plasma radii, increasing $d P / d V$, while it tends to reduce $d P / d V$ for plasma positions close to the magnetic axis due to a slightly higher overall reabsorption. As a general result, the variation of the net $\mathrm{EC}$ wave power loss with aspect ratio is small (less than $15 \%$ when comparing the results for large aspect ratio, i.e. the cylindrical limit, with those for low A).
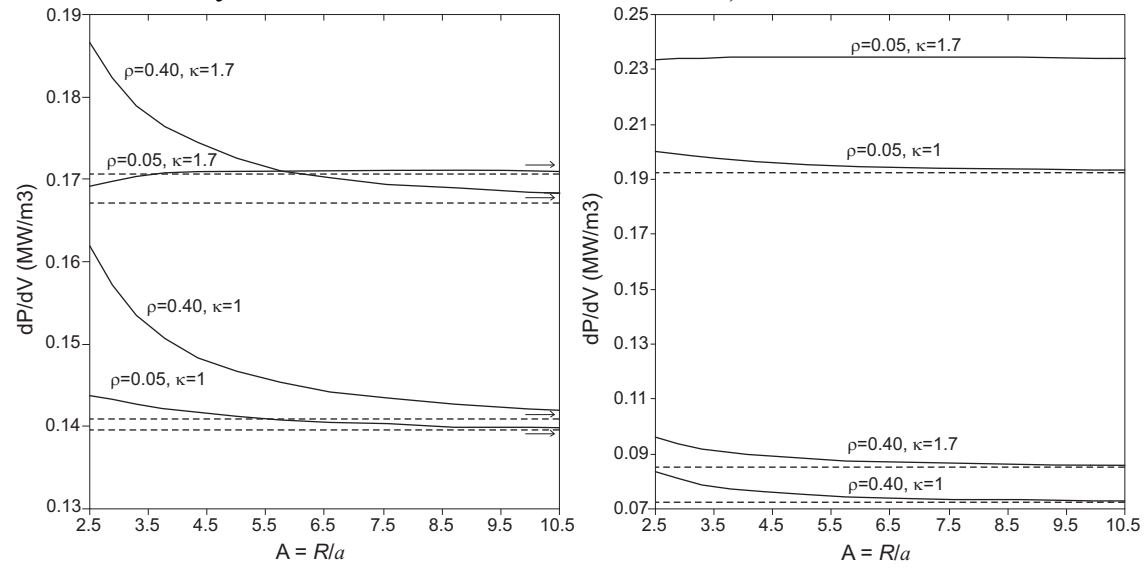

Figure 5. EC radiative power density $d P / d V$ versus aspect ratio $\mathrm{A}$ at $\rho=0.05$ and $\rho=0.40$, from RAYTEC for advanced (left plot) and parabola-like (right plot) temperature profiles: circular plasma cross-section $(\kappa=1)$ and $\kappa_{\text {ref }}=1.7$.

\section{Conclusions}

Electron cyclotron (EC) wave losses have been recognized to be important in the local electron power balance of reactor-grade tokamak plasmas with core temperatures of $35 \mathrm{keV}$ or higher as anticipated for steady-state operation in ITER and DEMO and, therefore, can influence the temperature profile of these plasmas. This has motivated an effort to improve the modelling capability of the radial profile of EC power losses. As part of this effort, recently the code RAYTEC has been set up for general toroidal geometry and for specular wall reflection. First results show that plasma elongation and toroidicity for ITERlike steady-state operating conditions, in the hot core, enhance these losses by no more than typically $25 \%$ with respect to an equivalent circularly cylindrical plasma. This is not far from what is found to occur just through isotropisation of 
the wave field, e.g., by a diffuse component in wall reflection ${ }^{11}$. Therefore, RAYTEC can be used with good accuracy (certainly considerably better than $25 \%$ locally) in 1D plasma transport calculations for ITER and DEMO. However, also the more simplified models underlying the computationally faster codes CYTRAN, CYNEQ and EXACTEC are quite adequate for this aim.

\section{References}

1. See,e.g., F. Albajar et al., "Importance of Electron Energy Transport in ITER", Nucl. Fusion 45, 642, 2005.

2. See, e.g., M. Bornatici, R. Cano, O. De Barbieri and F. Engelmann, "Electron Cyclotron Emission and Absorption in Fusion Plasmas", Nucl. Fusion 23, 1153, 1983.

3. F. Albajar, J. Johner and G. Granata, "Improved Calculation of Synchrotron Radiation Losses in Realistic Tokamak Plasmas", Nucl. Fusion 41, 665, 2001.

4. S. Tamor, "A Simple Fast Routine for Computation of Energy Transport by Synchrotron Radiation in Tokamaks and Similar Geometries", Report SAI023-81-189-LJ/LAPS-72 (La Jolla, CA: Science Applications), 1981.

5. A.B. Kukushkin, "Heat Transport by Cyclotron Waves in Plasmas with Strong Magnetic Field and Highly Reflecting Walls", Proc. 14th IAEA Conf. on Plasma Phys. and Contr. Nucl. Fusion, v.2, pp. 35-45, 1992.

6. B.A. Trubnikov, "Universal Coefficients for Syncrhrotron Emission from Plasma Configurations", Rev. Plasma Phys. vol 7 ed M A Leontovich (New York: Consultants Bureau), p.345, 1979.

7. P.A. Robinson, "Synchrotron Emission and Absorption by Relativistic Loss Cone and Anti-Loss Cone Distributions", Plasma Phys. Control. Fusion 27, 1037, 1985.

8. F. Albajar, N. Bertelli, M. Bornatici and F. Engelmann, "Electron Cyclotron Absorption in High-Temperature Plasmas: Quasi-Exact Analytical Evaluation and Comparative Numerical Analysis", Plasma Phys. Control. Fusion 49, 15, 2007.

9. F. Albajar, M. Bornatici and F. Engelmann, "Electron Cyclotron Wave Power Loss in Fusion Plasmas: a Model Comparison", Nucl. Fusion 47, 1101, 2007.

10. F. Albajar, M. Bornatici and F. Engelmann, "Electron Cyclotron Radiative Transfer in Fusion Plasmas", Nucl. Fusion 42, 670, 2002.

11. F. Albajar, M. Bornatici, F. Engelmann and A.B. Kukushkin, "Benchmarking of Codes for Calculating Local Net EC Power Losses in Fusion Plasmas", this Workshop, contributed paper.

12. S. Tamor, "Calculation of Energy Transport by Cyclotron Radiation in Fusion Plasmas”, Nucl. Technol. Fusion 3, 293, 1983. 